\title{
IJIMI
}

International Journal of Machine Intelligence

ISSN: 0975-2927 \& E-ISSN: 0975-9166, Volume 3, Issue 1, 2011, pp-18-24

Available online at http://www.bioinfo.in/contents.php?id=31

\section{QUADRATIC NUAT B-SPLINE CURVES WITH MULTIPLE SHAPE PARAMETERS}

\section{MRIDULA DUBE ${ }^{1}$ AND REENU SHARMA ${ }^{2}$}

${ }^{1}$ Department of Mathematics and Computer Science, R. D. University, Jabalpur

2Department of Mathematics, Mata Gujri Mahila Mahavidyalaya, Jabalpur

*Corresponding author. E-mail: reenusharma6@rediffmail.com

Received: April 15, 2011; Accepted: May 06, 2011

\begin{abstract}
In this paper a new kind of splines, called quadratic non-uniform algebraic trigonometric B-splines (quadratic NUAT Bsplines) with multiple shape parameters are constructed over the space spanned by $\left\{1, t, t^{2}, \sin t, \cos , \sin ^{2} t, \cos ^{2} t\right\}$. As each piece of the curves is generated by three consecutive control points, they possess many properties of the quadratic B-spline curves and quadratic trigonometric B-spline curves. These curves have $C^{1}$ continuity with non-uniform knot vector. These curves are closer to the control polygon than the quadratic B-spline curves and quadratic trigonometric B-spline curves when choosing special shape parameters. The shape parameters serve to local control in the curves. The changes of one shape parameter will only affect two curve segments. Taking different values of the shape parameters, one can globally or locally adjust the shapes of the curves. The generation of tensor product surfaces by these new splines is straightforward. Keywords- Quadratic NUAT B-spline; shape parameter; continuity; spline surface.
\end{abstract}

\section{Introduction}

The trigonometric B-splines were presented in [12]. The recurrence relation for the trigonometric B-splines of arbitrary order was established in [8]. The construction of exponential tension B-splines of arbitrary order was given in [7]. It was further shown in [13] that the trigonometric Bsplines of odd order form a partition of a constant in the case of equidistant knots.

In recent years, several bases in new spaces other than the polynomial space have been proposed for geometric modeling in CAGD. For instance, in [10] a basis is constructed for $\mathrm{C}_{\mathrm{m}}=\operatorname{span}\{1, \operatorname{cost}, \ldots, \cos m t\}$. In [11] a basis for the space of trigonometric polynomials \{ 1$, sint, cost,$\ldots$, sinmt, cosmt $\}$ is constructed. Some bases are constructed in [9] for the spaces \{ $1, t, \cos t, \sin t, \cos 2 t, \sin 2 t\},\left\{1, t, t^{2}\right.$, cost, sint $\}$ and \{ $1, t$, cost, sint, tcost, $t$ sint $\}$. In [1] C-Bézier curves are constructed in the space spanned by $\left\{1, t, t^{2}, \ldots, t^{n-2}\right.$, sint, cost $\}$. Non-uniform algebraic trigonometric B-splines (NUAT B-splines), are generated in [14] over the space spanned by $\left\{1, t, \ldots, t^{k-3}\right.$, cost, sint $\}$ in which $\mathrm{k}$ is an arbitrary integer larger than or equal to 3 . But all these curves do not have any shape parameter. In $[15,16] \mathrm{C}$-B-splines are presented in the space spanned by $\{1, t$, sint, cost $\}$ with parameter $\alpha$.
In order to improve the shape of the curve and adjust the extent to which a curve approaches its control polygon, some trigonometric curves are presented by using global shape parameters in $[2,5,6]$. In $[3,4]$ quadratic trigonometric polynomial curves with local shape parameters are constructed. A new kind of B-splines, quadratic non-uniform algebraic trigonometric (NUAT) Bsplines with multiple shape parameters, is presented in this paper. The present paper is organized as follows. In section 2, the basis functions with multiple shape parameters of the Quadratic NUAT B-Spline Curves are established and the properties of the basis functions are shown. In section 3, Quadratic NUAT B-Spline Curves are given. Continuity of the curves, Open and closed trigonometric, and shape control of the curves are discussed. In section 4, the corresponding Quadratic NUAT Bézier Curves with multiple shape parameters are defined and their properties are given. The Quadratic NUAT BSpline Curve approaches to the control polygon by increasing the shape parameters. It is shown in section 5 that the quadratic non-uniform algebraic trigonometric (NUAT) B-splines are closer to the control polygon than the quadratic algebraic B-spline curves, cubic algebraic Bspline curves and the quadratic trigonometric polynomial curves of [5] when choosing special shape parameters. The corresponding quadratic non-uniform algebraic trigonometric (NUAT) B-spline surfaces with multiple shape 
parameters are defined in section 6 . Our results are supported by various numerical examples in each section.

\section{Quadratic NUAT Basis Functions}

2.1 The construction of the basis functions Definition1. Given Knots $u_{0}<u_{1}<u_{2}<\ldots \ldots .<u_{n+3}$ and refer to $\mathrm{U}=\left(u_{0}, u_{1}, u_{2}, \ldots \ldots . u_{n+3}\right)$ as a knot vector. For shape parameters $\lambda_{i} \in[-2, a]$, where $a=1+2\left(\frac{2}{\pi}\right)^{2} \quad, \quad$ let $\quad \Delta u_{i}=u_{i+1}-u_{i}$ $\alpha_{i}=\frac{\Delta u_{i}}{\Delta u_{i-1}+\Delta u_{i}} \quad, \quad \beta_{i}=\frac{\Delta u_{i}}{\Delta u_{i}+\Delta u_{i+1}}$ $t_{i}(u)=\frac{\pi}{2}\left(\frac{u-u_{i}}{\Delta u_{i}}\right)$

$c_{i}(t)=\frac{1}{2}\left\{(1-\sin t)\left(1-\lambda_{i-1} \sin t\right)+\left(1-\frac{2}{\pi} t\right)^{2}\right\}$

$d_{i}(t)=\frac{1}{2}\left\{(1-\cos t)\left(1-\lambda_{i} \cos t\right)+\left(\frac{2}{\pi} t\right)^{2}\right\}$

Then the associated quadratic NUAT basis functions with multiple shape parameters are defined to be the following functions:

$b_{i}(u)=$

$$
\left\{\begin{array}{l}
\beta_{i} d_{i}\left(t_{i}(u)\right), \text { if } u \in\left[u_{i}, u_{i+1}\right), \\
1-\alpha_{i+1} c_{i+1}\left(t_{i+1}(u)\right)-\beta_{i+1} d_{i+1}\left(t_{i+1}(u)\right), \\
\text { if } u \in\left[u_{i+1}, u_{i+2}\right), \\
\alpha_{i+2} c_{i+2}\left(t_{i+2}(u)\right), \text { if } u \in\left[u_{i+2}, u_{i+3}\right) \\
0, \text { if } u \notin\left[u_{i}, u_{i+3}\right) .
\end{array}\right.
$$

For $\mathrm{i}=0,1,2, \ldots \ldots, \mathrm{n}$.

The properties of the quadratic NUAT basis functions Theorem 1 The basis functions (1) have the following properties:

(a) Non negativity : $b_{i}(u) \geq 0$ for $u_{i}<u<u_{i+3}$

(b) Partition of unity: $\sum_{i=0}^{n} b_{i}(u)=1, u \in\left[u_{2}, u_{n+1}\right]$

(c) Smallest support property: $\quad b_{i}(u)=0$ for $u_{0} \leq u \leq u_{i}, \quad u_{i+3} \leq u \leq u_{n+3}$ (d) Monotonicity: For $u \in\left[u_{i}, u_{i+1}\right), \quad \mathrm{i}=2,3, \ldots . . . \mathrm{n}$; $b_{i-2}(u)=\alpha_{i} c\left(t_{i}\right)$

$b_{i-1}(u)=1-\alpha_{i} c\left(t_{i}\right)-\beta_{i} d\left(t_{i}\right)$

$b_{i}(u)=\beta_{i} d\left(t_{i}\right)$

For a given value of knot $u \in\left[u_{i}, u_{i+1}\right), b_{i-2}(u)$ and $b_{i}(u)$ are monotonically decreasing as shape parameters $\lambda_{i-1}$ and $\lambda_{i}$ increase respectively and $b_{i-1}(u)$ is monotonically increasing with the increase in shape parameters $\lambda_{i-1}$ and $\lambda_{i}$ respectively. In view of the properties (a)-(d) we say that the basis functions has a support on the interval $\left[u_{i}, u_{i+3}\right] \forall$ $\mathrm{i}=0,1,2, \ldots \ldots, \mathrm{n}$. For equidistant knots, we refer to the $b_{i}(u)$ as a uniform basis function. Fig. 1 shows the curves of uniform basis functions for all $\lambda_{i}=-1.8$ (blue lines), $\lambda_{i}=0$ (green lines), and $\lambda_{i}=1.8$ (red lines) for the knots $u_{0}=0, u_{1}=1, u_{2}=2, u_{3}=3, u_{4}=4$, $u_{5}=5, u_{6}=6$. The basis functions defined over nonequidistant knots are called non-uniform basis functions. Fig 2 shows the curves of non-uniform basis functions for all $\lambda_{i}=-1.8$ (blue lines), $\lambda_{i}=0$ (green lines), and $\lambda_{i}=1.8$ (red lines) for the knots $u_{0}=0, u_{1}=0.7$, $u_{2}=1.5, u_{3}=2.2, u_{4}=5, u_{5}=6.3, u_{6}=7$. The basis functions defined over equidistant knots are called uniform basis functions.

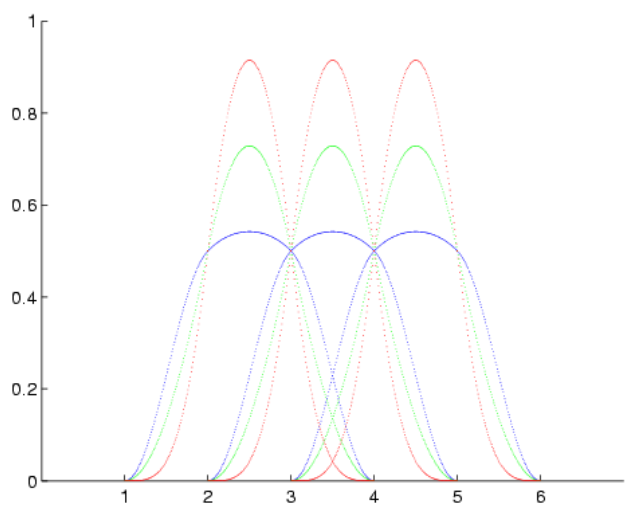

Fig. 1

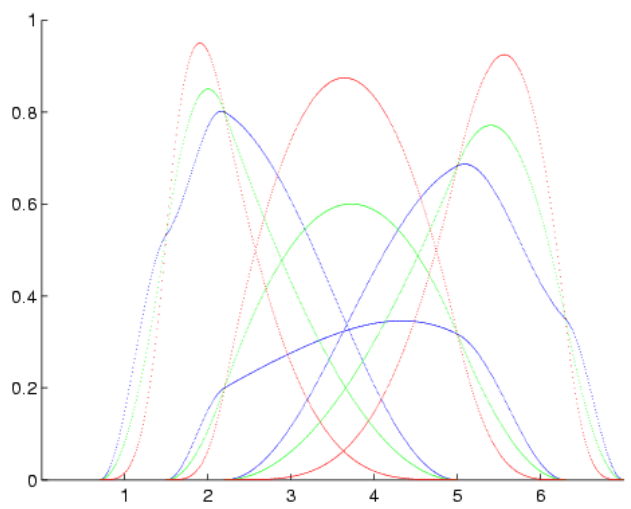

Fig. 2

\section{The continuity of the quadratic NUAT basis functions}

Theorem 2 The quadratic NUAT basis functions $b_{i}(u)$ has $C^{1}$ continuity at each of the knots.

Proof- Consider the continuity at the knots $u_{i+1}$ and $u_{i+2}$ (we can deal with the knots $u_{i}$ and $u_{i+3}$ in the same way). Straightforward computation gives that

$$
\begin{array}{ll}
b_{i}\left(u_{i+1}^{-}\right) & =\beta_{i} \quad b_{i}\left(u_{i+1}^{+}\right)=1-\alpha_{i+1} \\
b_{i}\left(u_{i+2}^{-}\right) & =1-\beta_{i+1} \quad b_{i}\left(u_{i+2}^{+}\right)=\alpha_{i+2} \\
b_{i}^{\prime}\left(u_{i+1}^{-}\right) & =\frac{\beta_{i}}{\Delta u_{i}}\left\{\frac{\pi}{4}\left(1+\lambda_{i}\right)+1\right\}, \\
b_{i}^{\prime}\left(u_{i+1}^{+}\right) & =\frac{\alpha_{i+1}}{\Delta u_{i+1}}\left\{\frac{\pi}{4}\left(1+\lambda_{i}\right)+1\right\}
\end{array}
$$


$b_{i}^{\prime}\left(u_{i+2}^{-}\right)=-\frac{\beta_{i+1}}{\Delta u_{i+1}}\left\{\frac{\pi}{4}\left(1+\lambda_{i+1}\right)+1\right\}$,

$b_{i}^{\prime}\left(u_{i+2}^{+}\right)=-\frac{\alpha_{i+2}}{\Delta u_{i+2}}\left\{\frac{\pi}{4}\left(1+\lambda_{i+1}\right)+1\right\}$

Since $\alpha_{j+1}=1-\beta_{j}, \frac{\alpha_{j+1}}{\Delta u_{j+1}}=\frac{\beta_{j}}{\Delta u_{j}}$,

$(0 \leq j \leq n+1)$, we get $b_{i}^{(k)}\left(u_{i+1}^{-}\right)=b_{i}^{(k)}\left(u_{i+1}^{+}\right)$

and $b_{i}^{(k)}\left(u_{i+2}^{-}\right)=b_{i}^{(k)}\left(u_{i+2}^{+}\right), \mathrm{k}=0,1$

The theorem follows.

\section{The case of multiple knots}

So far in the discussion of the basis functions, we have assumed that each point is simple. On the other hand, the basis functions also make sense when knots are considered with multiplicity $k<3$. For the quadratic NUAT basis functions, with multiple knots, it is worth notice that we shrink the corresponding intervals to zero and drop the corresponding pieces. For example if $u_{i+1}=u_{i+2}$ is a double knot, then we define

$b_{i}(u)=$

$\left\{\begin{array}{l}\beta_{i} d_{i}\left(t_{i}(u)\right), \text { if } u \in\left[u_{i}, u_{i+1}\right), \\ \alpha_{i+2} c_{i+2}\left(t_{i+2}(u)\right), \text { if } u \in\left[u_{i+2}, u_{i+3}\right), \\ 0, \text { if } u \in\left[u_{i}, u_{i+3}\right) .\end{array}\right.$

Theorem 3 Suppose that a basis function has a knot of multiplicity $k$ ( $k=2$ or 3 ) at the parameter value $u$. Then at this point the continuity of the basis function is reduced from $C^{1}$ to $C^{2-k}\left(C^{-1}\right.$ means discontinuity). Moreover the support interval of the basis function is reduced from 3 segments to $4-k$ segments.

Proof It is direct application of (1) and the expressions given in the proof of Theorem 2.

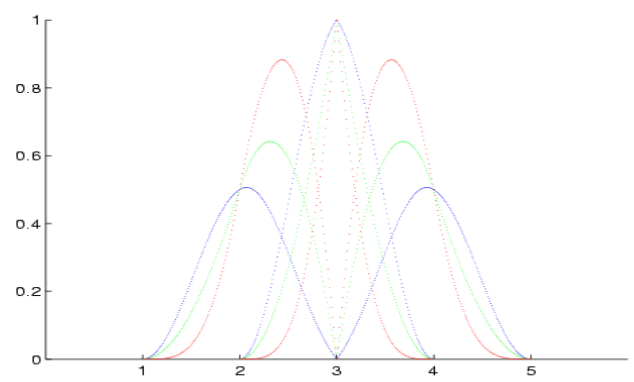

Fig. 3

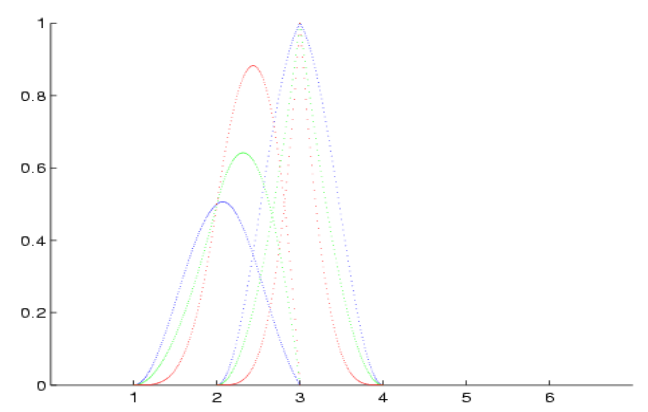

Fig. 4

Fig 3 shows the curves of the quadratic NUAT basis functions for all $\lambda_{i}=-1.8$ (blue lines), $\lambda_{i}=0$ (green lines), and $\lambda_{i}=1.8$ (red lines) with a double knot ( $u_{0}=0, u_{1}=1, u_{2}=2, u_{3}=3, u_{4}=3, u_{5}=4$, $u_{6}=5$ ). Fig 4 shows the curves of the quadratic NUAT basis functions for all $\lambda_{i}=-1.8$ (blue lines), $\lambda_{i}=0$ (green lines), and $\lambda_{i}=1.8$ (red lines) with a triple knot ( $u_{0}=0, u_{1}=1, u_{2}=2, u_{3}=3, u_{4}=3, u_{5}=3$, $\left.u_{6}=4\right)$.

\section{Quadratic NUAT Curves}

Definition 2. Given points $P_{i}(\mathrm{i}=0,1,2, \ldots, \mathrm{n})$ in $R^{2}$ or $R^{3}$ and a knot vector $\mathrm{U}=\left(u_{0}, u_{1}, \ldots \ldots, u_{n+3}\right)$. Then

$T(u)=\sum_{j=0}^{n} b_{j}(u) P_{j}$,

$n \geq 2, u \in\left[u_{2}, u_{n+1}\right]$,

is called a quadratic NUAT curve with multiple shape parameters.

Obviously, for $u \in\left[u_{i}, u_{i+1}\right)(2 \leq i \leq n)$ the curve $\mathrm{T}(\mathrm{u})$ can be represented by curve segment

$T(u)=b_{i-2}(u) P_{i-2}+b_{i-1}(u) P_{i-1}+b_{i}(u) P_{i}$

These curves have many properties of the quadratic Bspline algebraic curves and trigonometric curves of $[2,3,4$, $5,6]$, such as geometric invariance, convex hull property, symmetry, variation diminishing and locality.

Moreover,

$T\left(u_{i}^{+}\right)=\alpha_{i}\left(P_{i-2}-P_{i-1}\right)+P_{i-1}$

$T\left(u_{i}^{-}\right)=\beta_{i-1}\left(P_{i-1}-P_{i-2}\right)+P_{i-2}=\alpha_{i}\left(P_{i-2}-\right.$ $\left.P_{i-1}\right)+P_{i-1}$

$T^{\prime}\left(u_{i}^{+}\right)=\frac{\alpha_{i}}{\Delta u_{i}}\left\{\frac{\pi}{4}\left(1+\lambda_{i-1}\right)+1\right\}\left(P_{i-1}-P_{i-2}\right)$

$T^{\prime}\left(u_{i}^{-}\right)=\frac{\beta_{i-1}}{\Delta u_{i-1}}\left\{\frac{\pi}{4}\left(1+\lambda_{i-1}\right)+1\right\}\left(P_{i-1}-P_{i-2}\right)$

The continuity of the quadratic NUAT curves Analogous to the quadratic B-spline curves, the choice of the knot vector automatically determines the continuity of the quadratic NUAT curves at each of the knots as shown by the following theorem. 
Theorem 4 If a knot $u_{i}$ has multiplicity $k$ ( $\mathrm{k}=1,2$ or 3 ) then the quadratic NUAT curves have $C^{2-k}$ continuity. Proof It is a direct result of Theorem 2 and Theorem 3.

Open and closed quadratic NUAT curves Since the curve $T(u)$ is generated on the interval [ $\left.u_{2}, u_{n+1}\right]$, the choice of the first and last two knots is free and these knots can be adjusted to give the desired boundary behavior of the curve. See the following descriptions.

For an open algebraic trigonometric curve, we choose the knot vector $\mathrm{U}=\left(u_{0}=u_{1}=u_{2}, u_{3}, \ldots \ldots, u_{n}, u_{n+1}=u_{n+2}=\right.$ $\left.u_{n+3}\right)$. This assure that the points $P_{0}$ and $P_{n}$ are points on the curve. Of course, the interior knots can be multiple knots. Fig. 5 shows open NUAT curves for all $\lambda_{i}=-1.8,0$, 1.8 (red lines) respectively, open trigonometric curves of [5] for $\lambda_{i}=-1,0,1$ (blue lines), and the quadratic B-spline curves (green lines) corresponding to the same control polygon for a non uniform knot vector $u_{0}=u_{1}=u_{2}=3$, $u_{3}=5, u_{4}=5.5, u_{5}=8, u_{6}=9, u_{7}=10$, $u_{8}=u_{9}=u_{10}=11$. As the shape parameters $\lambda_{i}$ increases, the algebraic trigonometric curves (red lines) approach to the edge of control polygon. In order to construct closed quadratic algebraic trigonometric curves, we can extend the given points $P_{0}, P_{1}, P_{2}, \ldots \ldots, P_{n}$ by setting $P_{n+1}=P_{0}$, $P_{n+2}=P_{1}$ and let $\Delta u_{2}=\Delta u_{n+3}, \Delta u_{1}=\Delta u_{n+2}$ (such that $\left.T\left(u_{2}\right)=T\left(u_{n+3}\right), T^{\prime}\left(u_{2}\right)=T^{\prime}\left(u_{n+3}\right)\right)$, $u_{n+5} \geq u_{n+4}$. Thus the parametric formula for a closed quadratic NUAT curve is

$T(u)=\sum_{j=0}^{n+2} b_{j}(u) P_{j} \quad u \in\left[u_{2}, u_{n+3}\right]$ where $b_{n+1}(u)$ and $b_{n+2}(u)$ are given by expanding (1).

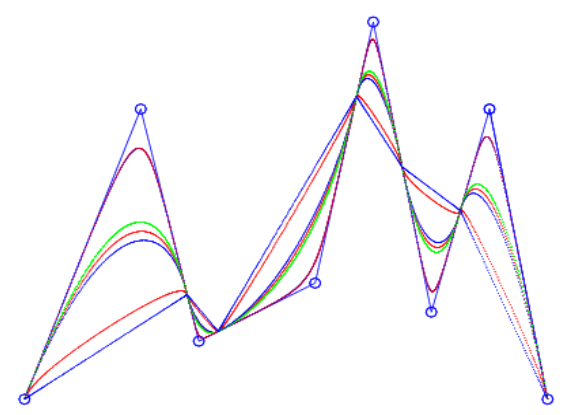

Fig. 5

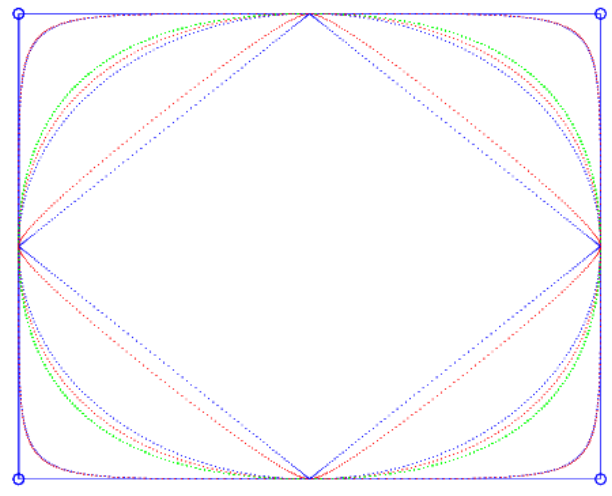

Fig. 6

Fig. 6 shows closed algebraic trigonometric curves for all $\lambda_{i}=-1.8,0,1.8$ (red lines) respectively, closed trigonometric curves of [5] for $\lambda_{i}=-1,0,1$ (blue lines), and the quadratic B-spline curves (green lines) corresponding to the same control polygon for a uniform knot vector.

\section{Shape control of the curves}

For $u \in\left[u_{i}, u_{i+1}\right], t_{i}=\frac{\pi\left(u-u_{i}\right)}{2 \Delta u_{i}}$ we rewrite (4) as follows

$T(u)=b_{i-2}(u) P_{i-2}+b_{i-1}(u) P_{i-1}+b_{i}(u) P_{i}$

$=\sum_{j=0}^{2} P_{i+j-2} r_{j}\left(t_{i}\right)+$

$\left(P_{i-2}-P_{i-1}\right) \frac{\alpha_{i}}{2} \lambda_{i-1} \sin t_{i}\left(\sin t_{i}-1\right)+$

$\left(P_{i}-P_{i-1}\right) \frac{\beta_{i}}{2} \lambda_{i} \cos t_{i}\left(\cos t_{i}-1\right)$

Where

$r_{0}\left(t_{i}\right)=\frac{\alpha_{i}}{2}\left\{1-\sin t_{i}+\left(1-\frac{2}{\pi} t\right)^{2}\right\}$

$r_{2}\left(t_{i}\right)=\frac{\beta_{i}}{2}\left\{1-\cos t_{i}+\left(\frac{2}{\pi} t\right)^{2}\right\}$

$r_{1}\left(t_{i}\right)=1-r_{0}\left(t_{i}\right)-r_{2}\left(t_{i}\right)$

Obviously, shape parameters $\lambda_{i-1}$ and $\lambda_{i}$ only affect curves on the control edge $\left(P_{i-2}-P_{i-1}\right)$ and $\left(P_{i}-P_{i-1}\right)$ respectively for all $i=2,3, \ldots, n+1$. From (5) we can also predict the following behavior of the curves. The curve has local control due to small support of $b_{i}(u)$. From (5) we can also predict the following behavior of the curves. Change of one control point will alter at most three segments of the curve. So local adjustment can be made without disturbing the rest of the curve. The shape parameters $\lambda_{i}$ also serve to effect local control in the curves. As $\lambda_{i-1}$ increases, the curve moves in the direction of the edge $\left(P_{i-2}-P_{i-1}\right)$ and as $\lambda_{i-1}$ decreases the curve moves in the opposite direction to the edge $\left(P_{i-2}-P_{i-1}\right)$ for all $i=2,3, \ldots, n+1$. As the shape parameter $\lambda_{i-1}=\lambda_{i}$ the curve moves in the direction of or the opposite direction to the control point $P_{i-1}$, when $\lambda_{i-1}\left(=\lambda_{i}\right)$ increases or decreases for all 
$i=2,3, \ldots, n+1$. Table 1 shows some computed examples with different values of shape parameters $\lambda_{i}$. These curves are generated by setting $\lambda=(1.8,1.8,1.8,1.8$, $1.8,1.8,1.8,1.8,1.8,1.8,1.8)$ in (a), $\lambda=(-1.5,-0.2,-1.8$, $1.8,-1.5,-1,-1.5,1.8,-1.8,-0.2,-1.5)$ in (b), $\lambda=(0,0,0,0$, $-1.8,0,-1.8,0,0,0,0)$ in (c), $\lambda=(1.8,1.8,-1.4,-1.8,1,1.5$, $1,-1.8,-1.4,1.8,1.8)$ in (d), $\lambda=(-1,-1.4,1.8,1.8,0,-1.5,0$, $1.8,1.8,-1.4,-1)$ in $(e), \lambda=(1.8,1.8,0.5,-1.5,-1.5,-1.5$, $1.5,-1.5,0.5,1.8,1.8)$ in (f), $\lambda=(1,1.2,1,-1.3,-1.5,1.8$, $1.5,-1.3,1,1.2,1)$ in $(\mathrm{g}), \lambda=(-1,-1.8,-1.65,-1.5,-1.51 .8,-$ $1.5,-1.5,-1.65,-1.8,-1)$ in $(h), \lambda=(1.8,1.8,-1.8,1.8,1.8$, $1.8,1.8,1.8,-1.8,1.8,1.8)$ in (i), $\lambda=(-1.8,1.8,-1.8,-1.8$, $1.8,1.8,1.8,-1.8,-1.8,1.8,-1.8)$ in (j).

\section{Quadratic NUAT Bézier curve with multiple shape parameters}

Let $u_{i}<u_{i+1}, u_{i}$ and $u_{i+1}$ be double points $\left(u_{i}\right.$ is a triple point when $\mathrm{i}=2$ and $u_{i+1}$ is a triple point when $\mathrm{i}=\mathrm{n}$ ), thus we have $\Delta u_{i-1}=\Delta u_{i+1}=0$, so we obtain the Quadratic NUAT Bézier basis functions with multiple shape parameters $\lambda_{i}$

$b_{i-2}(u)=\frac{1}{2}\left\{(1-\sin t)\left(1-\lambda_{i-1} \sin t\right)+(1-\right.$ $\left.\left.\frac{2}{\pi} t\right)^{2}\right\}$

$b_{i}(u)=\frac{1}{2}\left\{(1-\cos t)\left(1-\lambda_{i} \cos t\right)+\left(\frac{2}{\pi} t\right)^{2}\right\}$

$b_{i-1}(u)=1-b_{i-2}(u)-b_{i}(u) \quad, \quad$ where

$u \in\left[u_{i}, u_{i+1}\right], t=\frac{\pi\left(u-u_{i}\right)}{2 \Delta u_{i}}$

These basis functions have the following properties:

(1) $\sum_{j=i-2}^{i} b_{j}(u)=1$, for $u \in\left[u_{i}, u_{i+1}\right)$,

(2) For $u \in\left[u_{i}, u_{i+1}\right)$, if $\lambda_{i} \in[-2, a]$,

where $a=1+2\left(\frac{2}{\pi}\right)^{2}$,

then $b_{j}(u)>0 \quad(j=i-2, i-1, i)$

For the corresponding quadratic NUAT curve (4) in this case, we have

$T\left(u_{i}\right)=P_{i-2} \quad, \quad T\left(u_{i+1}\right)=P_{i} \quad$, $T^{\prime}\left(u_{i}\right)=\frac{1}{\Delta u_{i}}\left\{\frac{\pi}{4}\left(1+\lambda_{i-1}\right)+1\right\}\left(P_{i-1}-P_{i-2}\right)$

$T^{\prime}\left(u_{i+1}\right)=\frac{1}{\Delta u_{i}}\left\{\frac{\pi}{4}\left(1+\lambda_{i}\right)+1\right\}\left(P_{i}-P_{i-1}\right)$

We call the corresponding quadratic NUAT curve (4) as Quadratic NUAT Bézier curve with multiple shape parameters.

\section{Approximability}

Control polygon provides an important tool in geometric modeling. It is an advantage if the curve being modeled tends to preserve the shape of its control polygon.Now we show the relations of the quadratic NUAT curves with the B-spline curves of degree $d(d=2,3)$ and the quadratic trigonometric curves [5] corresponding to their control polygon.

Given data points $P_{i} \in R^{2}$ or $R^{3}(\mathrm{i}=0,1,2, \ldots . ., \mathrm{n})$ and knots $\left.u_{0}<u_{1}<u_{2}<\ldots .<u_{n+3}\right)$. For $u \in$ $\left[u_{i}, u_{i+1}\right]$, the associated B-spline curve of degree $\mathrm{d}(\mathrm{d}=2$ and 3 ) can be given by

$B_{i}(s)=a_{i 0}(s) P_{i-2}+a_{i 1}(s) P_{i-1}+a_{i 2}(s) P_{i}(6)$

Where $\mathrm{s}=\frac{\left(u-u_{i}\right)}{\Delta u_{i}}$

$a_{i 0}(s)=\alpha_{i}(1-s)^{d}$

$a_{i 1}(s)=1-\alpha_{i}(1-s)^{d}-\beta_{i} s^{d}$

$a_{i 2}(s)=\beta_{i} s^{d}$

With a non-uniform knot vector $U$, it is easy to show that $T_{i}\left(u_{i}\right)=B_{i}\left(u_{i}\right)$ for $\mathrm{i}=2,3, \ldots \ldots, \mathrm{n}+1$. From (4) we obtain

$T(u)-P_{i-1}=b_{i-2}(u)\left(P_{i-2}-P_{i-1}\right)+b_{i}(u)\left(P_{i}-\right.$ $\left.P_{i-1}\right)$

then taking the norm we obtain

$\left\|T(u)-P_{i-1}\right\| \leq\left(b_{i-2}(u)+b_{i}(u)\right) \max (\|$ $\left.P_{i-2}-P_{i-1}\|,\| P_{i}-P_{i-1} \|\right)$

Since $b_{i-2}(u)$ and $b_{i}(u)$ decreases as $\lambda_{i-1}$ and $\lambda_{i}$ increases, from (7) we know that quadratic NUAT curve segments approach to their control polygon with the increase of $\lambda_{i-1}$ and $\lambda_{i}$.

$b_{i-2}(u)+b_{i}(u)$ get its minimum value at $u=\frac{u_{i}+u_{i+1}}{2}$ (i.e. $t_{i}=\frac{\pi}{4}$ ) and

$T\left(\frac{u_{i}+u_{i+1}}{2}\right)-P_{i-1}=\frac{\alpha_{i}}{2}\left\{\left(1-\frac{1}{\sqrt{2}}\right)\left(1-\frac{\lambda_{i-1}}{\sqrt{2}}\right)+\right.$

$\left.\frac{1}{4}\right\}\left(P_{i-2}-P_{i-1}\right)+$

$\frac{\beta_{i}}{2}\left\{\left(1-\frac{1}{\sqrt{2}}\right)\left(1-\frac{\lambda_{i}}{\sqrt{2}}\right)+\frac{1}{4}\right\}\left(P_{i}-P_{i-1}\right) \quad$ at

$\lambda_{i-1}=\lambda_{i}=\lambda \forall i=0,1, \ldots, n$, we have

$T\left(\frac{u_{i}+u_{i+1}}{2}\right)-P_{i-1}=\frac{1}{4}[(\sqrt{2}-1)(\sqrt{2}-\lambda)+$ $\left.\frac{1}{2}\right]\left[\alpha_{i} P_{i-2}-\left(\alpha_{i}+\beta_{i}\right) P_{i-1}+\beta_{i} P_{i}\right]$

For the associated Quadratic B-spline curve ( $d=2$ ) segment $B_{i}(s)$ we have $B_{i}\left(\frac{1}{2}\right)-P_{i-1}=\frac{1}{4}\left[\alpha_{i} P_{i-2}-\left(\alpha_{i}+\beta_{i}\right) P_{i-1}+\beta_{i} P_{i}\right]$

Let $\quad \frac{1}{4}\left[(\sqrt{2}-1)(\sqrt{2}-\lambda)+\frac{1}{2}\right]=\frac{1}{4} \quad, \quad$ then $\lambda=-0.20710$. From here we know that Quadratic NUAT curves are closer to the control polygons than the Quadratic B-spline curves when $\lambda>-0.20710$. Similarly we can show that Quadratic NUAT curves are closer to the control polygons than the - (i) cubic B-spline curves ( $d=3$ ) when $\lambda>1.4142$ and (ii) Quadratic trigonometric curves of [5] when $\lambda>1.7927$

\section{The Quadratic NUAT Surfaces}

Given control points $P_{i j}(\mathrm{i}=0,1,2, \ldots, \mathrm{m} ; \mathrm{j}=0,1,2, \ldots, \mathrm{n})$ and the knot vectors $U=\left(u_{0}, u_{1}, u_{2}, \ldots ., u_{m+3}\right)$ and $V=\left(v_{0}, v_{1}, v_{2}, \ldots ., v_{n+3}\right)$, using the tensor product method, we can construct quadratic NUAT surface $T(u, v)=\sum_{i=0}^{m} \sum_{j=0}^{n} b_{i}\left(\lambda_{1, i}, u\right) b_{j}\left(\lambda_{2, j}, v\right) P_{i j}$; $m, n \geq 2 ; u \in\left[u_{2}, u_{m+1}\right], v \in\left[v_{2}, v_{n+1}\right]$ 
where $b_{i}\left(\lambda_{1, i}, u\right)$ and $b_{j}\left(\lambda_{2, j}, v\right)$ are the quadratic NUAT basis functions with multiple shape parameters $\lambda_{1, i}$ and $\lambda_{2, j}$ respectively. Obviously these surfaces have properties similar to the corresponding quadratic NUAT curves. This surface is $C^{1}$ continuous. In addition, since the surfaces have multiple shape parameters, the shape of the surfaces can be adjusted from two direction (in each direction using multiple shape parameters), so they can more conveniently be used in the outline design.

\section{References}

[1] Chen Q., Wang G. (2003) Computer Aided Geometric Design 20, 29-39.

[2] Han X. (2004) Computer Aided Geometric Design, 21, 535-548.

[3] Han X., (2005) Journal of Computational and Applied Mathematics, 180, 161-172.

[4] Han X. (2006) Applied Numerical Mathematics, $56,105-115$.

[5] Han X. (2002) Computer Aided Geometric Design, 19, 479-502.

[6] Han X.A., Ma Y.C., Huang X.L. (2009) Applied Mathematical Letters, 22, 226-231.

[7] Koch P.E., Lyche T. (1991) Construction of exponential tension $B$-splines of arbitrary order.
In: Laurent, P.J., Le Méhauté, A., Schumaker, L.L. (Eds.), Curves and Surfaces. Academic Press, NewYork, , 255--258.

[8] Lyche T., Winther R. (1979) J. Approx. Theory 25, 266-279.

[9] Mainar E., Peña J.M., Sânchez-Reyes J. (2001) Computer Aided Geometric Design, 18, , 37-60.

[10] Peña J.M. (1997) Computer Aided Geometric Design, 14, , 5-11.

[11] Sânchez-Reyes J. (1998) Computer Aided Geometric Design, 15, 909-923.

[12] Schoenberg I. J. (1964) J.Math. Mech. 13 795825.

[13] Walz G. (1997) BIT 37 (1), 189-201.

[14] Wang G., Chen Q., Zhou M. (2004) Computer Aided Geometric Design, 21, 193-205.

[15] Zhang J.W. (1996) Computer Aided Geometric Design, 13, 199-217.

[16] Zhang J.W., (1997) Computer Aided Geometric Design, 14, 31-41. 
Table 1- Some computed examples with different values of shape parameters $\lambda_{i}$

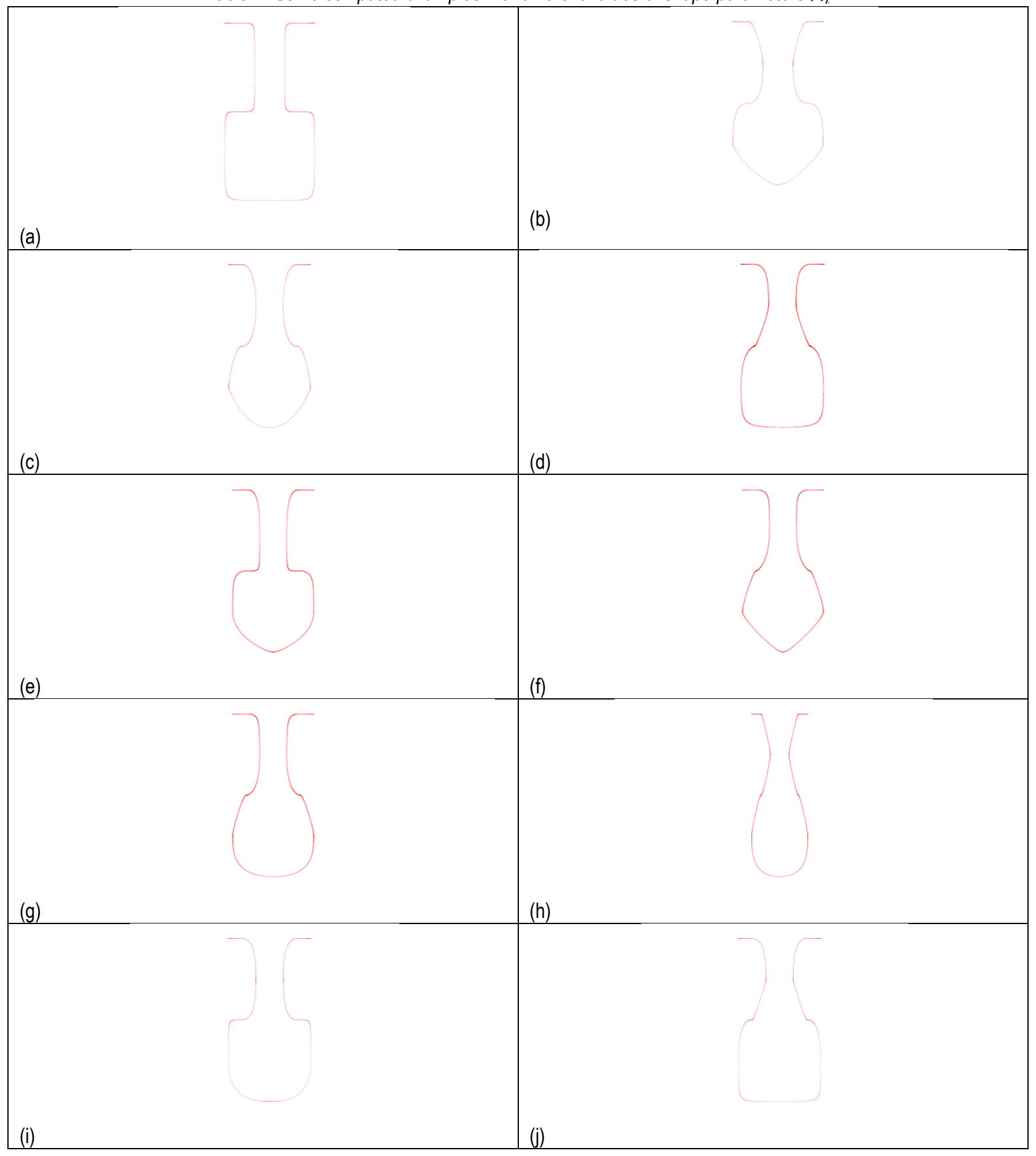

